\title{
THE DOPPLER EFFECT AND THE SPEED OF LIGHT DO NOT CHANGE
}

\author{
Duan Xianxiang
}

Summary:The assumption that the speed of light does not change contradicts the doppler effect. In the real universe, the speed of light is not a constant speed between the light source and the observer. The speed of light is relative and time is absolute.

Keywords: invariant speed of light, doppler effect, absolute velocity, relative velocity, absolute time

The nature of time

\section{1 nature of time}

Einstein's special theory of relativity has always maintained that "the speed of light does not change," that is:

In two relatively uniformly moving reference systems, the light waves emitted by the light source of one reference system are still at the speed of light in the other reference system, and Einstein did so by modifying the time of the reference system.

However, this paper argues that in the real world, the time of two reference frames is the same, and the time of reference frames cannot be modified. Time is an abstract concept. Like the Arabic numerals "1, 2, 3, 4, 5", it is an abstract existence. It has no physical substance. Time is defined by human beings, and if the time of reference frame is modified, the calculation will become meaningless.

This is like:

$1+3=4$

This is true. But if someone thinks: "1=2", and then substitute this "1=2" into the previous formula, namely:

$1+3=2+3=5$

That's not consistent with the previous result. This is the definition of changing the number "1", changing the definition of "1" to "2", and then taking it back to the original formula.

Special relativity does the same thing, Special relativity works the same way, modifying the time, and then bringing it into the original computing system.

However, such a modification of time would lead to confusion in the definition of time, making calculations with "time" physical quantities meaningless.

So, in the universe, all the reference systems have the same time, time is not relative, anywhere in the universe, in any space, time goes by the same way, because time doesn't exist, and its meaning is defined by man. Man defines time as "the same everywhere in the universe", that is, time is absolute, and this is the essence of time. 1.2 the clock

A clock is not the same thing as time. The clock is not an abstract concept defined by human beings. The clock is real, it is not an abstract concept, it has physical entities. Therefore, it can be affected by physical factors, such as gravitational field. 
In the time experiment, the time didn't change, the clock did. GPS time goes faster because the atomic clock goes faster, and the atomic clock goes faster because it oscillates at a higher frequency, because of the gravitational field of the earth.

The intensity of gravitational field on the earth's surface and the upper air has different influence on the oscillation frequency of atoms. The oscillation frequency of atoms is high in the upper air and low in the ground. This is an atomic clock, a physical entity that has changed, but time is not a physical entity, Atomic clocks are just timing tools, It's not time. Time is the unit that defines the speed of change, the same as the unit of length defined in meters and inches. You can't change the definition of "meter," .

If a person uses his finger to move the pointer of the watch and changes the watch's reading, can he say that he has changed the time?

The gravitational field is the finger, and it changes the reading of the atomic clock.

Reason for GPS clock becomes fast.

Two gravitational fields and characteristics have been played a role in this phenomenon. One is that the intensity of gravitational field is related to the distance of gravitation source, which is the characteristic of gravitation in above (4). The distance of satellite to the gravitation source of earth is further than the distance of clock on the ground to earth. The gravitation of the location of satellite is much less and the oscillation frequency of atom of atomic clock on the satellite is higher. In addition, the movement of satellite generates the torsion through the characteristic of gravitation in (5). But because it is the circle movement with low velocity, the effect of torsion is little. Therefore, the characteristic of gravitation in (4) plays a role in general and the atomic clock of satellite is faster than that in the ground.

All in all, gravitational field can change the motion trajectory of the object in field; and it can change the oscillation frequency of particle. The oscillation frequency of atomic clock is changed, but the time is unchanged. The gravitational field change the time of a clock is essentially the same with that human adjusts a watch. Human adjusts the time, which does not change the time, namely: clock $\neq$ time. ${ }^{[1]}$

\section{The speed of light is not absolute}

There should be a relative velocity between the velocity of the light wave emitted by the light source and its own velocity, that is, the speed of light cannot be superimposed. The theory of special relativity holds that the speed of light relative to the source itself is still the speed of light $c$, The theory of special relativity holds that 
the speed of light relative to the source itself is still the speed of light. However, the theory of special relativity holds that the speed of light cannot be superimposed with the speed of the light source. That is, the speed of light cannot be superimposed, nor can it be combined with the speed of the light source.

For example, if you turn on a flashlight on a train, The speed of the light emitted by the flashlight is not "the speed of light $c \pm$ the speed of a train.". In the eyes of observers at rest on the ground, the speed of light waves is still the speed of light.

That is to say, there is a relative speed between the light wave of a flashlight and the train, In the train reference system, the speed of light of a flashlight is relative.

But the theory of special relativity holds that the speed between the light wave of the flashlight and the train is still $c$, that is, "the speed of light does not change.". It is also believed that the speed of flashlight measured by a stationary observer on the ground is also $c$, It is also believed that the velocity of light waves emitted by a flashlight measured by a stationary observer on the ground is also $c$. That is, the speed between the light wave and the flashlight is $c$, and the velocity between the light wave and the ground observer is $c$.

If a medium is needed for the transmission of light waves, it is for the same reason that sound waves cannot be superimposed. The velocity of sound waves through the air is independent of the motion of the sound source. When a wave crest leaves the source, it is traveling in a medium, and its velocity is independent of the source's motion. So in a frame of reference, like a flashlight on a train, The speed of light and the speed of a train cannot be superimposed. Of course, for the observer who is stationary on the ground, the speed of the light wave is still the speed of light $c$, because the observer on the ground is stationary relative to the light wave medium, then the relative speed of the light wave is the speed of the medium relative to the light wave, namely the speed of light $c$.

Let's assume that the velocity between the light wave and the light source itself is relative, and consider the time of the light source reference system and the observer reference system to be the same. And then derive the doppler effect formula to see if it holds.

2.1 reference frame of relatively uniform motion

In fact, the best example of two reference systems with relatively uniform motion is the "Doppler effect". In the Doppler effect, the light source and the observed person are regarded as two reference systems of constant velocity relative motion.In this case, if Einstein's "invariant speed of light hypothesis" is used, the doppler effect cannot be established.

2.2 as can be seen from the wavelength formula:

$$
f=\frac{v}{\lambda} \text { (1) }
$$

In the formula (1), the frequency of the light source is constant, its own motion will not affect its light frequency. As a result, $v$ and $\lambda$ Changes need to be synchronized, that is, when calculating the frequency of the light source, the wave speed and 
wavelength need to be regarded as changing or unchanged at the same time.

In the doppler effect:

$f^{\prime}=\left(\frac{v \pm v_{0}}{v \mp v_{s}}\right) f$

$f^{\prime}$ Is the observed frequency;

$f$ The original emission frequency of the light source in the medium;

$v$ Is the traveling speed of the wave in the medium;

$v_{0}$ Is the moving speed of the observer. If it is close to the light source, the forward operation sign is + ; otherwise, it is -.

$v_{s}$ Is the moving speed of the light source; if it is close to the observer, the forward operation sign is -; otherwise, it is + .

The formula is derived as follows:

$f^{\prime}=\frac{v \pm v_{0}}{\lambda^{\prime}}$

$\lambda^{\prime}$ Is the wavelength of the wave received by the observer.

$f=\frac{v \mp v_{s}}{\lambda^{\prime \prime}}$

$\lambda^{\prime \prime}$ Is the wavelength of the light wave in the medium after the

light wave emitted by the light source.

As you can see, let formula (2) hold, " $\lambda^{\prime}=\lambda^{\prime \prime}$ " also must be true, that is, the wavelength of the optical wave received by the observer is the wavelength of the optical wave in the medium.

If the doppler effect formula is to be established, it must be:

When the light source is close to the observer, the wavelength of the light $\left(\lambda^{\prime \prime}\right)$ wave should be considered to get shorter because the frequency " $f$ " is constant, and its wave speed " $v \mp v_{s} "$ should also be seen as get smaller (according to the formula(1)). In this way, the doppler effect formula (4) is established.

According to the doppler effect, the frequency of a source is calculated by treating both wavelength and velocity as change, or just as constant. Either the wavelength and the speed of the waves are seen as changing, or both are seen as being constant, and not just one or the other.

In the light source reference frame, the formula of the speed " $v \mp v_{s}$ " should be seen as the relative velocity, is the relative velocity between the light wave and the source.

When the observer is close to the light source, in formula (3), the wavelength of the light " $\lambda$ " " wave remains unchanged, but the frequency becomes higher, and the 
wave speed $" v \pm v_{0}$ " is increases, otherwise the formula cannot be established.

In other words, the relative velocity between the observer and the light wave should also be calculated, instead of using the speed of light $c$ directly, otherwise the doppler effect formula will not hold.

From the above we can see that the doppler effect formula can be derived by regarding the speed of light as the relative speed.

2.3 when the speed of light is absolute

However, according to the relativistic view, when the light source is moving, the speed of the light wave relative to the source in the reference frame is still the speed of light $c$.That is to say: the speed of light is the absolute speed of the universe, which is a constant relative to any reference system, even if it is relative to the light source itself is a constant relative $c$.

$v \mp v_{s}=c$ (5)

Similarly, in the formula, according to the relativistic point of view, there is:

$v \pm v_{0}=c$

Substitute (5) and (6) into (5) and (4), and get:

$f^{\prime}=\frac{c}{\lambda^{\prime}}$

$f=\frac{c}{\lambda^{\prime \prime}}$

This obviously does not lead to a formula for the doppler effect.

Moreover, in formula (3) and (4), " $v$ " is the velocity of wave in medium, and for light wave, is the speed of light $c$, that is:

$v=c$ (7)

But relativity says that the speed of light waves relative to the observer is still "c". Relativity says:

$v \pm v_{0}=c$ This, however, contradicts the (7).

Of course, the frequency of the light source can also be calculated as follows:

$f=\frac{v}{\lambda}$

" $v$ " Is the velocity of a wave in this medium, " $\lambda$ " is the wavelength of the light source when it is not moving. There are:

$$
f=\frac{v}{\lambda}=\frac{v \mp v_{s}}{\lambda^{\prime \prime}}
$$

That is to say, the frequency of the light source is the same as that of the stationary source, but it is difficult to deduce the Doppler formula with the formula of 
(8) instead of the formula (4). In the end, it needs to be replaced by (4) to derive the formula (2).

3 The assumption that the speed of light will change

The paper posits a hypothesis contrary to special relativity, and argues that this hypothesis is a fact of the real world:

The speed of light is not always constant with respect to the light source and the observer " $c$ ".

So let's say on the surface of the earth, we have A light source, A, and we have an observer, A, who emits blue light.

The observer measures the speed of light when both the light source A and the observer A are stationary " $c$ ". This is because, when both the light source and the observer are stationary, not only the blue light waves, but the light waves of any frequency, the observer's measured speed of light is constant " $c$ ".

However, as the observer of A moves towards the light source, the light wave seen by the observer will shift purple (but the light source itself is still blue), because the frequency is getting higher and the blue light moves towards the purple light. However, if the observer measured the wavelength using Young's double slit interference principle, he still measured the wavelength of blue light. If he then used the formula (1) calculation:

$$
v=\lambda_{1} f_{2}
$$

Here, " $\lambda_{1}$ " is the wavelength of blue light, " $f_{2}$ " is the frequency of purple light, then the observer will calculate the speed of light is greater than constant " $c$ ". The observer measures the speed of light as the product of the wavelength of blue light and the frequency of purple light. That is:

When the observer moves, the wavelength he measures and the frequency he sees do not belong to the same color light waves, and the speed of light calculated is not "c".

Thus, it can be said that in the real world, the speed of light is not always constant with respect to the source and the observer " $c$ ".

4 Light waves of the same color may not have the same wavelength

Again, let's say on the surface of the earth, we have two light sources and two observers. A light source is stationary and emits blue light.

When the observer of A stands still, he measures wavelength " $\lambda_{\mathrm{A}}$ ".

The light source B is also stationary, and the color of the light source is unknown. The observer of B is moving at high speed towards the light source of B, and what he sees is the blue wave, which has the same frequency as the blue light source of $\mathrm{A}$ in front of him. However, the wavelength measured by B is not equal to the wavelength of blue light.

Because according to the formula (3), the frequency of the light the observer sees is: 


$$
f_{A}=\frac{v}{\lambda_{A}}
$$

B frequency of light seen by the observer:

$$
f_{B}=\frac{v+v_{0}}{\lambda_{B}}=f_{A}=\frac{v}{\lambda_{A}}
$$

It can be seen that to make the above equation true:

$$
\lambda_{B} \neq \lambda_{A}
$$

Therefore, the same red stars seen on earth may have the same frequency to people on earth, but they may not have the same wavelength, because the earth may be moving towards them at different speeds.

\section{Reference}

[1] Duan, XianXiang.Qualitative Description on Characteristics of GravitationaField[J]. OSF Preprints 10.31219/osj.io/x2vzf. 